\title{
Universal Linear Density of States for Tunneling into the Two-Dimensional Electron Gas in a Magnetic Field
}

\author{
H. B. Chan, P. I. Glicofridis, and R. C. Ashoori \\ Department of Physics, Massachusetts Institute of Technology, Cambridge, Massachusetts 02139 \\ M. R. Melloch \\ Department of Electrical Engineering, Purdue University, West Lafayette, Indiana 47907
}

(Received 10 February 1997)

\begin{abstract}
We have developed a technique capable of measuring the tunneling current into both localized and conducting states in a $2 \mathrm{D}$ electron system. $I-V$ characteristics for tunneling are obtained with no distortions arising from low 2D in-plane conductivity. In a perpendicular magnetic field, a pseudogap develops in the tunneling density of states at the Fermi energy. We resolve a linear energy dependence of this pseudogap at low excitations. The slopes of this linear gap are strongly field dependent. The data are suggestive of a new model of the gap in the presence of disorder and external screening. [S0031-9007(97)04269-5]
\end{abstract}

PACS numbers: 73.20.Dx, 71.45.Gm, 73.40.Gk

Characteristics of electrons tunneling into a twodimensional (2D) system differ considerably from those of the three-dimensional (3D) case. The distinction is especially pronounced when a magnetic field is applied in the tunneling direction (perpendicular to the plane in the $2 \mathrm{D}$ case). In the simplest picture, such a magnetic field effectively localizes electrons in the 2D system. An electron tunneling into an energetically unfavorable position cannot readily move away and instead tends to move in circles. As a result, tunneling measurements of 2D systems in a magnetic field display effects attributable to a "pseudogap" in the tunneling density of states (TDOS) at low injection energies [1-4]. In contrast, for 3D systems the tunneling electron can move parallel to the field lines to evade being localized at a position of high potential energy and such a field-induced gap has not been detected.

A number of experimental difficulties have impaired the study of electron tunneling into a 2D layer. First, in conventional tunneling measurements, electrons that tunnel into a 2D system introduce charge which must be removed from the system to avoid local charge accumulation at long time scales. This is achieved by conduction within the $2 \mathrm{D}$ plane. As a result, only contributions from delocalized states can be detected. The energetics of tunneling into localized sites, though of great theoretical interest, have not been measured experimentally. Second, previous schemes for probing the energetics of electron tunneling into $2 \mathrm{D}$ in semiconductors $[2,3]$ relied on the measurement of the tunneling current between two 2D layers, yielding a convolution of effects from both layers. Tunneling from a 2D into a spectroscopically featureless 3D layer achieves superior resolution of 2D features [5]. However, until now it was only possible to measure tunneling at zero bias between 2D and 3D in semiconductor heterostructures $[1,4]$.

Here, we introduce a new technique that we call "timedomain capacitance spectroscopy" (TDCS) for measuring the TDOS of a 2D electron system (2DES). TDCS eliminates the problems discussed above by measuring all of the current entering or exiting the 2D layer, including contributions arising from electrons entering localized sites. Using TDCS, we measure the tunneling current into a 2DES from a 3D system at arbitrarily low values of the 2D in-plane conductivity. We can now determine the complete $I-V$ characteristics for structures to which direct Ohmic contact is not possible.

In this Letter, we discuss results from a detailed survey of the excitation dependence of tunneling into a 2DES in a magnetic field and in the presence of disorder. In the low excitation regime, we find a behavior which is not predicted by any of the prior theoretical models for this system [6-8]. The TDOS is found to have a universal linear dependence on energy near the Fermi energy for all field strengths and electron densities. Moreover, the slopes of this linear gap are proportional to the inverse of the magnetic field strength over a wide range of fields.

Figure 1(a) depicts the type of samples used in our experiment. The 2DES is sandwiched between two electrodes, close enough only to the bottom electrode to permit tunneling of electrons. Mesas etched from two wafers grown using molecular beam epitaxy have been studied. Wafer $A$ consists of a degenerately $n$ doped $\left(10^{17} \mathrm{~cm}^{-3}\right)$ GaAs substrate followed by a $160 \AA$ AlGaAs tunnel barrier. On top of that, a $150 \AA$ GaAs quantum well is grown which defines the 2DES. A thick (1550 ̊) AlGaAs blocking barrier prevents charge transfer between the well and the top GaAs electrode. The blocking barrier contains an $n$ doped region to provide electrons for the well. Measurements were performed on circular mesas with $400 \mu \mathrm{m}$ diameters. This sample has been studied in detail previously using frequency dependent capacitance measurements [1] to determine zero-bias tunneling characteristics. Wafer $B$ [9] has a smaller dopant concentration in the blocking barrier and a thinner tunnel barrier $(143 \AA)$. A dc bias 


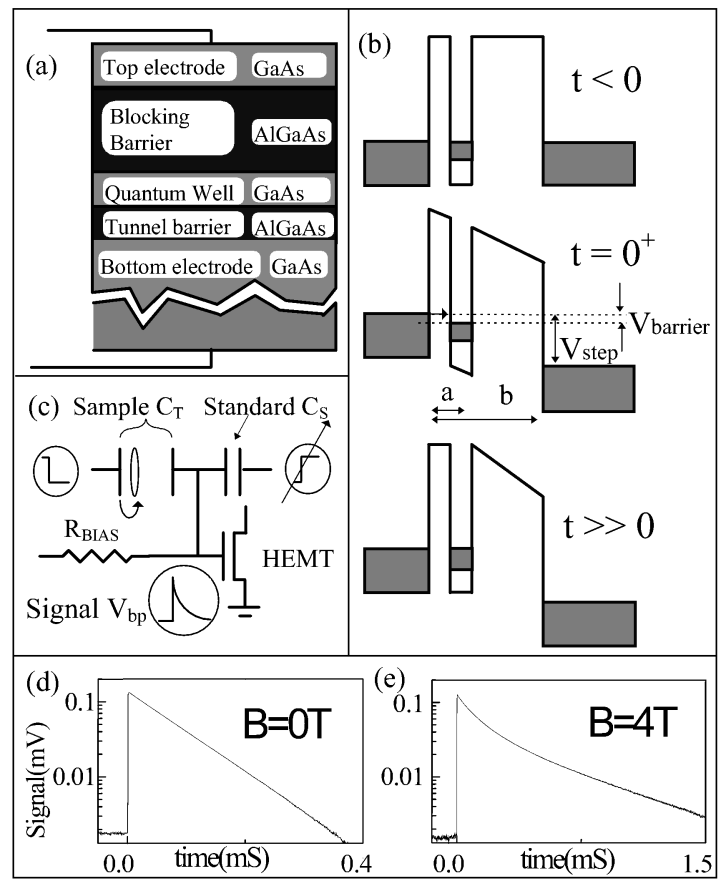

FIG. 1. (a) Structure of our samples. (b) Evolution of the conduction band profile of our sample in one measurement cycle. (c) External circuit used to measure the current flowing out of the sample. (d) Typical signal $\left(V_{\mathrm{bp}}\right)$ at the HEMT input for zero magnetic field, using $3.3 \mathrm{mV}$ excitation voltage steps. Notice the exponential decay with time. (e) At $4 \mathrm{~T}$, the recorded trace deviates from an exponential decay. The decay rate decreases as the signal decays.

applied to the top gate permits variation of the electron density in the quantum well from depletion to $6 \times 10^{11} \mathrm{~cm}^{-2}$ (sample $A$ ) and $3 \times 10^{11} \mathrm{~cm}^{-2}$ (sample $B$ ). The mobility of the 2DES in sample $A$ was estimated [10] to be $1 \times 10^{5} \mathrm{~cm}^{2} / \mathrm{V} \mathrm{s}$ at a density of $2 \times 10^{11} \mathrm{~cm}^{-2}$, and the mobility of sample $B$ is expected to be higher. All features of the tunneling suppression described in this paper have been observed in both samples.

Figure 1(b) shows the evolution of the conduction band diagram for our samples during one cycle of TDCS measurement. We start with the 2DES in equilibrium with the $3 \mathrm{D}$ substrate. At time $t=0$, a sharp $(<10 \mathrm{~ns}$ step rise) voltage step is applied. This creates an offset in the chemical potential on the two sides of the tunnel barrier, inducing a tunneling current. As electrons tunnel, this offset equilibrates, and the decay signal is recorded in real time. To measure the current across the tunnel barrier, a capacitance bridge [Fig. 1(c)] is utilized. Voltage steps of opposite polarity are applied to the substrate of the sample $\left(C_{T}\right)$ and to one plate of a standard capacitor $\left(C_{s}\right)$ at precisely the same instant. The other plate of $C_{s}$ and the top electrode of $C_{T}$ are electrically connected, and the signal at this balance point is fed into the gate of a high electron mobility transistor (HEMT).

Figures 1(d) and 1(e) display typical recorded signals, reflecting the voltage at the balance point of the bridge $\left(V_{\mathrm{bp}}\right)$ as a function of time. The electron tunneling current between the substrate and the 2D layer gives rise to a displacement current that charges $C_{s}$, leading to the observed decay signal. The amplitude of the step applied to $C_{s}$ is adjusted so that this signal decays to zero after electrons cease tunneling. If the tunneling conductance is independent of the voltage across the barrier, the recorded signal will be a familiar $R C$ exponential decay, as illustrated by the $\log$ scale plot in Fig. 1(d). On the other hand, a voltage dependent tunneling conductance, as in the case of magnetic field-induced tunneling suppression, will produce a decay which deviates from a pure exponential as in Fig. 1(e). The dc potential at the bridge balance point is fixed by a resistor $R_{\mathrm{BIAS}}(70 \mathrm{M} \Omega)$. Charge transfer through $R_{\mathrm{BIAS}}$ occurs on long time scales ( $>100 \mathrm{~ms})$ and does not distort the much faster observed tunneling signals. All measurements described in this Letter take place at a temperature of $30 \mathrm{mK}$.

Using charge conservation, we have determined that there is a fixed relationship between the barrier current and the displacement (measured) current. After application of the voltage step, the current across the barrier is found to be proportional to the initial time derivative of the voltage at the balance point:

$$
\begin{aligned}
I_{\mathrm{barrier}} & =-C_{\Sigma} \frac{d V_{\mathrm{bp}}}{d t} \text { where } \\
C_{\Sigma} & =\frac{C_{1} C_{S}+C_{2} C_{S}-C_{1} C_{2}}{C_{1}} .
\end{aligned}
$$

$C_{1}$ and $C_{2}$ in Eq. (1) are the simple geometrical capacitances $C_{1}=\epsilon A /(b-a)$ and $C_{2}=\epsilon A / a$, where $a$ and $b$ are defined in Fig. 1(b), $\epsilon$ is the dielectric constant, and $A$ is the area of the mesa. Elsewhere [11], we prove that relationship (1) remains true, independent of changes in the measured capacitance of the sample created by variations of thermodynamic DOS in the quantum well.

Before the voltage step is applied, the 2D plane has the same electrochemical potential at all positions. Immediately after the voltage step is applied $\left(t=0^{+}\right)$, no charge has been transferred into the 2DES, and the 2D plane remains an equipotential. The simple planar geometry of the sample dictates that this is true even in situations of very low 2D conductivity. At $t=0^{+}$, the voltage across the tunnel barrier $\left(V_{\text {barrier }}\right)$ is simply a fraction of the voltage step applied $\left(V_{\text {step }}\right)$, given by $V_{\text {barrier }}=(a / b) V_{\text {step }}$. The 3D substrate is always highly conducting, and electrons can tunnel everywhere into the 2DES, including localized tunneling sites.

By applying voltage steps of different amplitude and taking initial time derivatives of the corresponding transistor signal, the complete $I-V$ characteristics of the tunnel barrier can be mapped out. Signals from the experiment are extremely faint, and immense signal averaging is involved in our measurements. A novel signal processing [12] and rapid averaging system permits data acquisition with $\sim 18$ bit digital resolution.

Figure 2 shows the tunneling conductance $(I / V)$ of sample $A$ plotted against the voltage across the barrier 


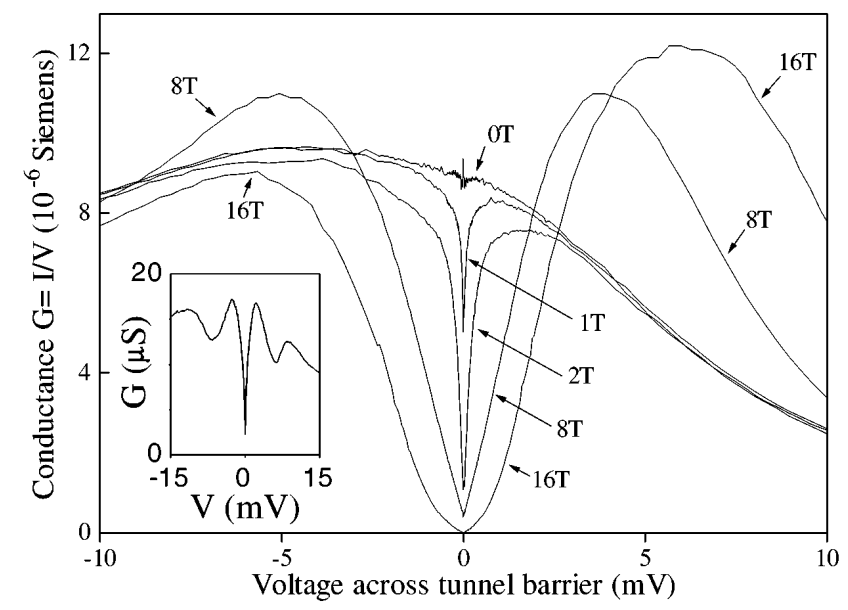

FIG. 2. Dependence of the tunneling conductance $(I / V)$ of sample $A$ on excitation voltage across the tunnel barrier for different magnetic field strengths at $30 \mathrm{mK}$ and a fixed density of $1.9 \times 10^{11} \mathrm{~cm}^{-2}$. Inset: conductance curve for sample $B$ at $4 \mathrm{~T}$ and $\nu \sim 3$.

for magnetic field strengths of $0,1,2,8$, and $16 \mathrm{~T}$ at a fixed electron density of $1.9 \times 10^{11} \mathrm{~cm}^{-2}$. This density is high enough so that no zero-bias tunneling suppression is observed at zero field. Application of a magnetic field reduces the tunneling conductance around zero bias. The suppression becomes deeper and wider as the field is increased. This field-induced tunneling suppression differs qualitatively from the logarithmic suppression [13] observed in the low density regime at zero field [11]. An increase in the strength of the suppression is accompanied by a change in the curvature of the high excitation part of the conductance curves when the magnetic field is increased. Even though the conductance curves at high excitation appear rounded at high fields, the zero-bias region remains linear in voltage, with both the magnitude and the slope significantly reduced. This singular behavior is illustrated by the insets of Fig. 3, which zoom in near the zero-bias region of the conductance curves for different field strengths. Such a linear energy dependence of the TDOS is observed over the full range of densities in both samples, except near depletion $\left(n \leq 5 \times 10^{10} \mathrm{~cm}^{-2}\right)$. The inset of Fig. 2 displays a conductance curve from sample $B$ at a field of $4 \mathrm{~T}$ and $\nu \sim 3$. In addition to the zero-bias suppression, features associated with adjacent Landau levels can be identified at higher excitations.

To our knowledge, no existing model other than the 2D Coulomb gap $[8,14]$ predicts linear DOS at low energies for such a wide range of field strengths and densities as observed in our samples. However, contrary to expectations for a Coulomb gap, the slopes of the observed linear gap are strongly field dependent. Figure 3 shows the slopes of the linear regions of the conductance curves plotted against inverse magnetic field. For filling factors $\nu<1$, the data points fall onto a straight line extrapolating to a negative intercept on the vertical axis. For low fields, there are de-

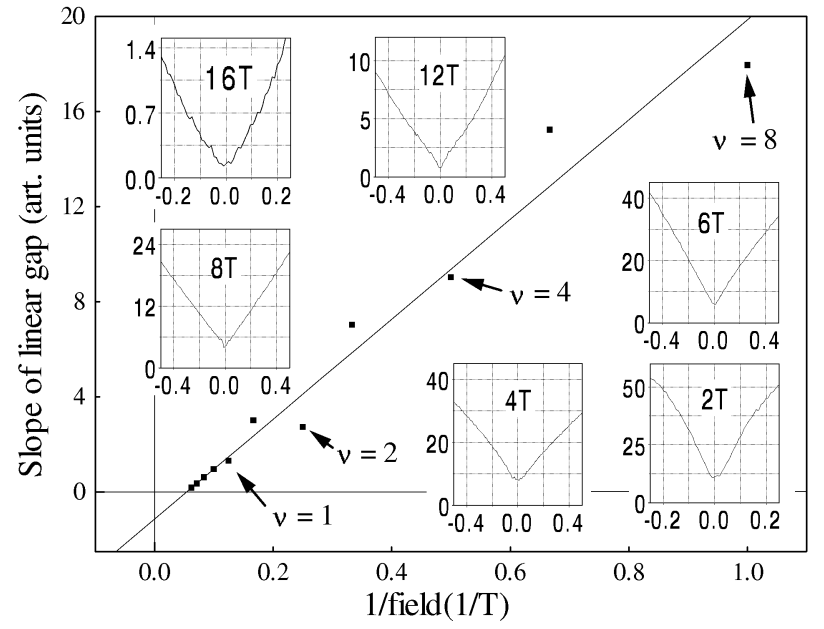

FIG. 3. Insets: tunneling conductance of sample $A$ vs excitation voltage for six different magnetic fields. The units are the same in all insets, with the abscissa in $\mathrm{mV}$ and the ordinates in microsiemens $(\mu \mathrm{S})$. Different ranges are chosen to display the singular behavior near zero bias. Main figure: dependence of the slope of this linear gap on inverse field strength.

viations from the straight line as $\nu$ varies between integer and noninteger values.

For different 2D electron densities, the conductance curves remain linear near zero bias. As a function of density, the slopes of the linear pseudogap exhibit minima at integer $\nu$. For instance, at $8 \mathrm{~T}$ the magnitude of the slope drops by a factor of 2 at $\nu=2$ compared to $\nu=1.5$. In Fig. 3, data points corresponding to integer $\nu$ are marked. These minima in the slope of the linear gap at integer $\nu$ might be attributed to a smaller background DOS between two Landau levels. Other than particulars associated with integer $\nu$, our data display only a very weak overall density dependence.

In the Coulomb gap picture, the states in the vicinity of the Fermi level are assumed to be localized. These electrons are treated as classical point charges at fixed positions in space with no overlap of the electronic wave functions. The phase space available for electron tunneling is reduced since it costs more energy to add an electron to the system when another electron is located close to the tunneling electron. The resulting Coulomb gap in the TDOS varies linearly with excitation energy in 2D with a slope determined solely by physical constants such as the electronic charge and dielectric constant [14]. In contrast, the slope of the linear gap in our data depends inversely on field strength with an offset, suggesting that our data cannot be explained by a simple Coulomb gap.

While no existing theory explains our results, the main features of our data are consistent with predictions from a simple phenomenological model. In this picture, the 2DES is modeled as isolated puddles with uniform charging energies and random background offsets. We note that the 2DES may indeed appear incompressible [6] at the very fast time scales for tunneling and thereby inhibit tunneling while still permitting $\mathrm{dc}$ transport in the $2 \mathrm{D}$ 


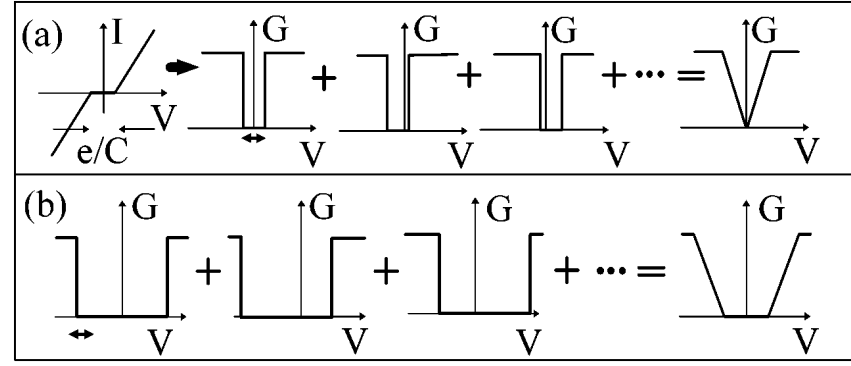

FIG. 4. (a) From the left: $I-V$ curve and corresponding conductance curve of a single puddle. A random offset voltage shifts the conductance curves along the voltage axis. Summation of these randomly shifted curves results in a Vshaped overall conductance curve. (b) When the puddles become small enough so that $e / C$ is larger than the background offset voltage, the overall conductance vanishes near zero bias.

plane. In our model, electrons tunnel into compressible puddles that exist within this incompressible background. In contrast to the Coulomb gap model, interactions among the puddles are neglected. This assumption may be justified by the presence of the nearby 3D electrode which screens the interactions among the puddles.

Each puddle contributes a Coulomb blockade type $I$ $V$ characteristic, leading to a conductance curve which is constant in voltage except for a region of width $e / C$ (where $C$ is the capacitance of the puddle) around zero bias where the conductance vanishes [Fig. 4(a)]. The conductance curve of an individual puddle is shifted from zero bias by a random background voltage offset created by nearby dopants or impurities. The sum of conductances from all puddles will thus be linear in voltage near zero bias and the slope of this linear gap is proportional to $C$. Since $C$ is proportional to the area of the puddles, our data can be explained if the average area of a puddle varies inversely with magnetic field.

This model can also explain the observation that the slopes of the linear gap extrapolate to zero at large but finite magnetic field (Fig. 3). In the high field limit, the puddles become small enough so that their Coulomb blockade energies exceed the range of the background offset energies. In this regime, summing contributions from different puddles leads to zero conductance at low bias up to a certain voltage beyond which the conductance rises linearly with voltage to the unsuppressed value, as depicted in Fig. 4(b). It is not necessary to have an infinitesimal puddle to achieve a zero slope for conductance near zero bias.

The above deductions are based on the assumptions that larger puddles break up into smaller ones and that the mean area of the puddles shrinks linearly with increasing field strength. Electrons therefore charge parallel plate capacitors whose lateral dimension is proportional to the magnetic length. From the width of the gap in our data, we estimate the proportionality constant to be about 6 . As the model fits our data significantly better than other existing models, we speculate that puddles may indeed form in the disordered 2D layer, although we do not know what the compressible puddles are or why they apparently shrink as the magnetic field is increased.

A preliminary TDCS study of a sample with much higher mobility $\left(>10^{6} \mathrm{~cm}^{2} / \mathrm{V} \mathrm{s}\right)[15]$ indicates that the low energy behavior of the tunneling suppression differs from that in the more disordered 2D systems discussed in this paper in two basic ways: (1) While the linear energy dependence is still observed for magnetic fields below 3 $\mathrm{T}$, the slopes of the $\mathrm{V}$-shaped pseudogap decrease more rapidly with increasing magnetic field. Above $3 \mathrm{~T}$, the gap becomes nonsingular and continues to widen as the field strength is raised. (2) At high fields, the zero-bias conductance is strongly suppressed, by 4 to 7 orders of magnitude from the zero field value, depending on the precise value of $\nu$.

In summary, we used a novel capacitance technique to measure the contributions of both localized and conducting tunneling states to the TDOS of a 2DES. In the presence of disorder and screening from a nearby $3 \mathrm{D}$ electrode, the suppression in the TDOS is found to be linear in energy near the Fermi energy for a wide range of densities and field strengths. The slope of this linear pseudogap varies inversely with magnetic field strength.

We thank L. S. Levitov, A. V. Shytov, J. K. Jain, R. K. Kamilla, and A.H. MacDonald for helpful discussions, and M. Brodsky for assistance in the experiment. This work is supported by the ONR, JSEP-DAAH04-951-0038, the Packard Foundation, NSF DMR-9357226, DMR-9311825, and DMR-9400415.

[1] R. C. Ashoori et al., Phys. Rev. Lett. 64, 681 (1990); R. C. Ashoori et al., Phys. Rev. B 48, 4616 (1993).

[2] J. P. Eisenstein, L. N. Pfeiffer, and K. W. West, Phys. Rev. Lett. 69, 3804 (1992).

[3] K. M. Brown et al., Phys. Rev. B 50, 15465 (1994).

[4] V.T. Dolgopolov et al., Phys. Rev. B 51, 7958 (1995).

[5] Y. Imry and Z. Ovadyahu, Phys. Rev. Lett. 49, 841 (1982); A. E. White, R.C. Dynes, and J.P. Garno, Phys. Rev. B 31, 1174 (1985).

[6] I. L. Aleiner, H. U. Baranger, and L. I. Glazman, Phys. Rev. Lett. 74, 3435 (1995); S. He, P. M. Platzman, and B. I. Halperin, Phys. Rev. Lett. 71, 777 (1993); P. Johansson and J. M. Kinaret, Phys. Rev. Lett. 71, 1435 (1993); S.-R.E. Yang and A.H. MacDonald, Phys. Rev. Lett. 70, 4110 (1993).

[7] A. A. Koulakov, M. M. Fogler, and B. I. Shklovskii, Phys. Rev. Lett. 76, 499 (1996).

[8] F. G. Pikus and A.L. Efros, Phys. Rev. B 51, 16871 (1995).

[9] Grown by M. L. Melloch.

[10] R. C. Ashoori and R. H. Silsbee, Solid State Commun. 81, 821 (1992).

[11] H. B. Chan et al. (to be published).

[12] R. C. Ashoori, U.S. Patent No. 5640124.

[13] B. L. Altshuler, A. G. Aronov, and P. A. Lee, Phys. Rev. Lett. 44, 1288 (1980).

[14] A. L. Efros, and B. I. Shklovskii, J. Phys. C 8, L49 (1975).

[15] Grown by L. N. Pffeifer and K. W. West. 